\title{
Chemical Synthesis of Copper Oxide Nanoparticles Study of its Optical and Electrical Properties
}

\author{
R. Chopra*, N. Kashyap ${ }^{1}$, Amit Kumar ${ }^{1}$, D. Banerjee ${ }^{2}$ \\ ${ }^{1}$ A Department of Mechanical Enginnering Roorkee College of Engineering, Roorkee, Uttrakhand, India, \\ ${ }^{2}$ Faculty of Engineering and Computer Science, Teerthanker Mahaveer University, \\ Moradabad, UP 244001
}

\begin{abstract}
A simple method for the synthesis of Nano-rod like copper oxide nanostructure has been reported using sodium hydroxide $(\mathrm{NaOH})$ and ultrapure deionized (DI) water and ethanol as precursor. Efforts were made to see effort of sodium hydroxide amount of different properties of the materials thus three sample were synthesized with different amount of sodium hydroxide precursors. The as-prepared sample was characterized by X-Ray diffraction (XRD), field emission scanning electron spectroscope study. Also the samples were characterized further with the help of Fourier-Transformed infrared (FTIR) spectroscopic study as well as Raman Spectroscopic study taken in reflection mode. XRD conforms the proper Phase formation of the sample whereas the microscopic analysis has given an idea about the morphological as well as dimensional information. FTIR study gives the information regarding the different bonding present in sample. Reflection study shows that, due to the change in the precursor amount, there is a marked difference in the reflection spectra as well as optical gap of the sample.
\end{abstract}

Keywords: Microscopic Study, Nano Structures, Copper Oxide, Optical Property, Electrical Property.

\section{INTRODUCTION}

Nano scale metal oxide materials have been attracting much attention because of their unique size- and dimensionalitydependent physical and chemical properties as well as promising applications as key components in micro/Nano scale devices. Cupric oxide $(\mathrm{CuO})$ nanostructures are of particular interest because of their interesting properties and promising applications in batteries, super capacitors, solar cells, gas sensors.

Copper oxide is a semiconductor material and has a natural abundance of starting material $(\mathrm{Cu})$. It is non-toxic and easily obtained by the oxidation of $\mathrm{Cu}$. Copper oxide is one of the important metal oxide which has attracted recent research because of its low cost, abundant availability as well as its peculiar properties. It is used in the fields like catalysis, superconductors, ceramics as a kind of important inorganic materials etc.

$\mathrm{CuO}$ has been used as a basic material as the superconductivity in these classes of systems is associated with $\mathrm{Cu}-\mathrm{O}$ bonding. Among all metal oxide nanoparticles, copper oxide has gained the most interest because of its wide applications, such as in solar cell technology, field emission, magnetic storage media, lithium ion batteries, gas sensing, drug delivery, magnetic resonance imaging, and field emission devices.

\section{Applications}

$\mathrm{CuO}$ first attracted attention of chemists as a good catalyst in organic reactions but recently discovered applications of $\mathrm{CuO}$ such as high-Tc superconductors, gas sensors, solar cells, emitters, electronic cathode materials also make this material a hot topic for physicists and materials science engineers. Some of the most interesting applications of $\mathrm{CuO}$ Nanomaterial's are sensing, photo catalyst, and super capacitor.

$\mathrm{CuO}$ Nano-materials have wide range of applications in the several fields i.e.

In very first huge application in electrical and electronics devices.

Super-capacitors and Electrodes for Lithium Ion Batteries Sensing Applications

Photo catalyst and Solar Energy Conversion Field Emission Effect [6]

\section{Materials}

\section{EXPERIMENTAL}

Copper chloride $(\mathrm{Cu})$, Sodium hydroxide $(\mathrm{NaOH})$ and Ultrapure Deionized (DI) water and ethanol. All materials were purchased and used without further purification.

\section{Synthesis procedure}

$\mathrm{CuO}$ nanostructure was synthesized by precipitation method using copper chloride $(\mathrm{Cu})$. First. Copper chloride was dissolved in $100 \mathrm{ml}$ deionized water to form $(0.1 \mathrm{M})$ concentration, $\mathrm{NaOH}$ solution $(0.1 \mathrm{M})$ was slowly dropped under vigorous stirring until $\mathrm{pH}$ reached to 10,12 and 14 respectively, So there are three different precursor ratio are taken i.e. $\mathrm{pH} 10, \mathrm{pH} 12$ and $\mathrm{pH} 14$. Black precipitates were obtained and repeatedly, washed by ultrapure deionized water and absolute ethanol sequentially added till $\mathrm{pH}$ reached [7]. Subsequently, the washed precipitates were dried at $80{ }^{\circ} \mathrm{C}$ for $16 \mathrm{~h}$. [13]

$$
\mathrm{Cucl}_{2}+2 \mathrm{NaOH} \rightarrow \mathrm{CuO}+2 \mathrm{Nacl}_{2}+\mathrm{H}_{2} \mathrm{O}
$$




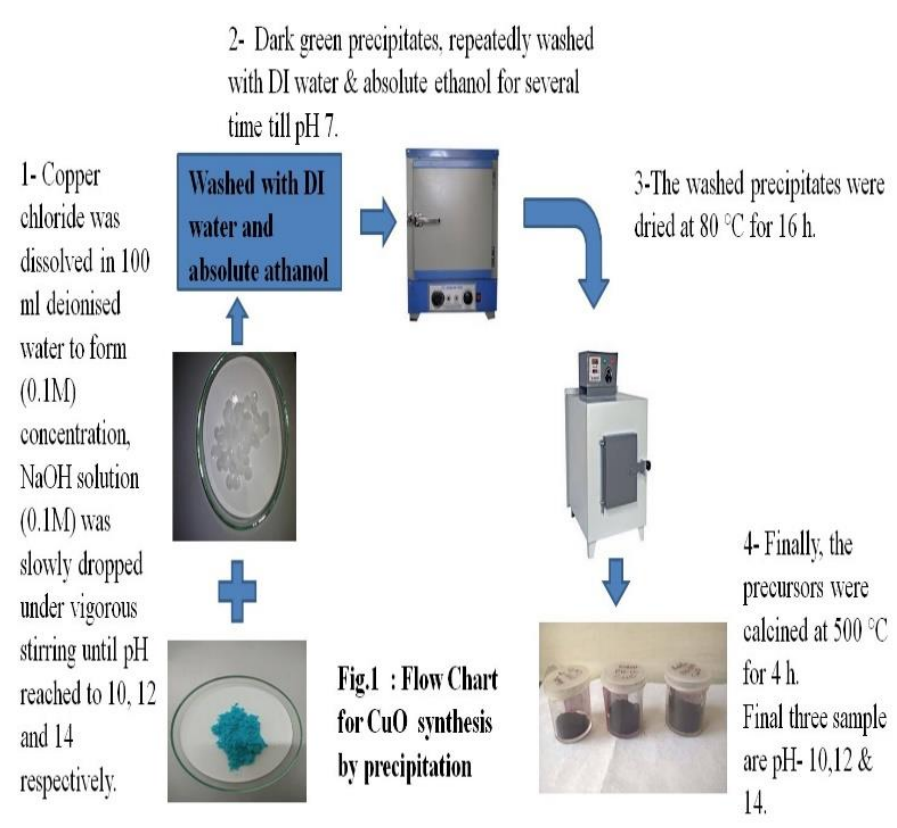

Finally, the precursors were calcined at $500{ }^{\circ} \mathrm{C}$ for $4 \mathrm{~h}$. investigated by X-Ray Diffractrometry (XRD). The morphology was monitored by Fourier transformation scanning electron microscope (FESEM). Chemical properties were investigated by Fourier transform infrared spectroscopy and the optical properties were obtained by the Raman spectroscope.

\section{RESULTS AND DISCUSSIONS}

The as prepared samples were characterized by X-ray diffraction (XRD Bruker, D8 Advance, $\mathrm{Cu} \mathrm{K}_{\alpha}$ Radiation), field emission scanning electron microscope (FESEM, Hitachi, S-4800). FTIR spectrophotometer (Shimadzu FTIR-8400S)

\section{Characterizations \\ $X R D$ analysis}

Fig. 2 shows the XRD pattern of the $\mathrm{CuO}$ powder prepared by precipitation method.

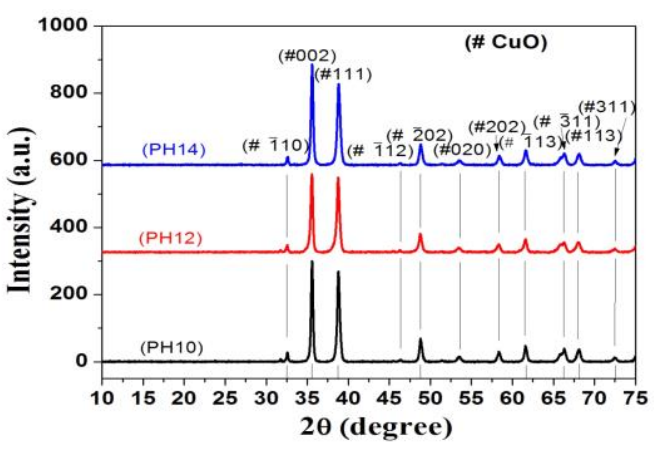

Fig 2 XRD patterns of $\mathrm{CuO}$ powder

The phase evolution and microstructure were characterized by X-ray diffraction method taken between $10^{\circ}-70^{\circ}$ with normal -2 scanning to determine the phase and crystallinity of the powder samples.
All the diffraction peaks in the pattern can be indexed to a single phase monoclinic structure was obtained. [3,18] No peaks of impurities other than $\mathrm{CuO}$ were detected, which are indicate high purity of synthesized $\mathrm{CuO}$. The sharp peaks indicate the well-crystallized single phase $\mathrm{CuO}$ Nano crystals. The highly diffracted peak is observed at angle $=35.54$ corresponding to the (002) lattice orientation. [15]

The various all other peaks i.e. (111), (020) and (202) are observed for $\mathrm{CuO}$ Nano rods. The broadening of the XRD peaks provides a convenient method for the measurement of average crystallite size. The average crystallite size was calculated by using Scherrer's formula,

$$
\tau=k \lambda / \beta \cos 2 \theta
$$

Where, is average crystallite size or grain size, $\mathrm{k}$ is shape factor (usually taken as 0.89 but at this time taken is 0.89 ), $\lambda$ is the wavelength of the X-ray beam used $(\lambda=0.154 \mathrm{~nm})$, is width at half maximum (FWHM) and is the angle of diffraction.

The grain sizes we have obtained from the scherrer formula these three are $3.1 \mathrm{~nm}, 3.2 \mathrm{~nm}$ and $3.4 \mathrm{~nm}$ at the angles $(2 \theta)$ of $35.53^{\circ}, 38.83^{\circ}$ and $48.81^{0}$ respectively. So that the average particle size is $23 \mathrm{~nm}$ for $\mathrm{CuO}$ polyclinic structure.

\section{SEM Study}

Scanning electron microscopy is proved to be a unique method to analyze the morphological properties of nanostructure.

Fig 3 shows the FESEM image of $\mathrm{CuO}$ morphology of the $\mathrm{CuO}$ is Nano rods. Morphological properties have a strong influence on the optical properties of the nanostructure. It can be observed that the ethanol have substantial impact on the growth of $\mathrm{CuO}$ synthesized using $\mathrm{NaOH}$ as a complex agent showed the formation of $\mathrm{CuO}$ nanostructure like rod.

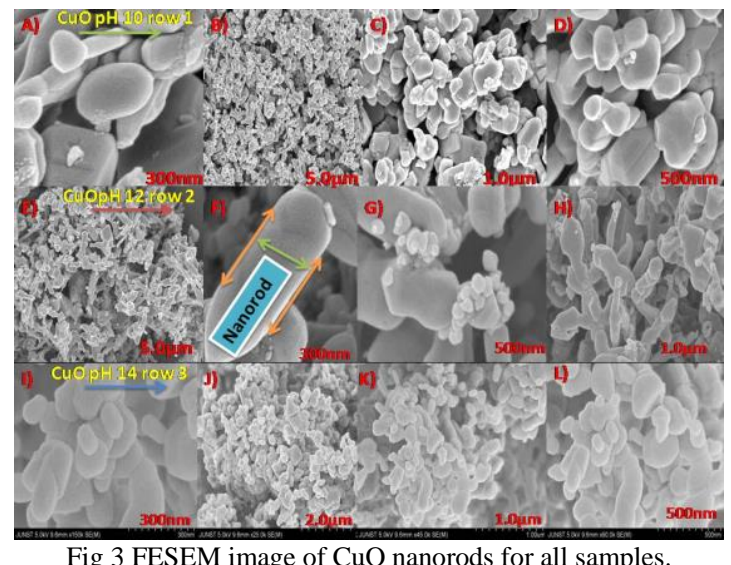

The morphological properties have a strong influence on the optical properties of the nanostructure.

After calcinations at 500 product of $\mathrm{CuO}$ tends to agglomerate with increasing rod size and clustered form clearly occurred in the product prepared.[1] 


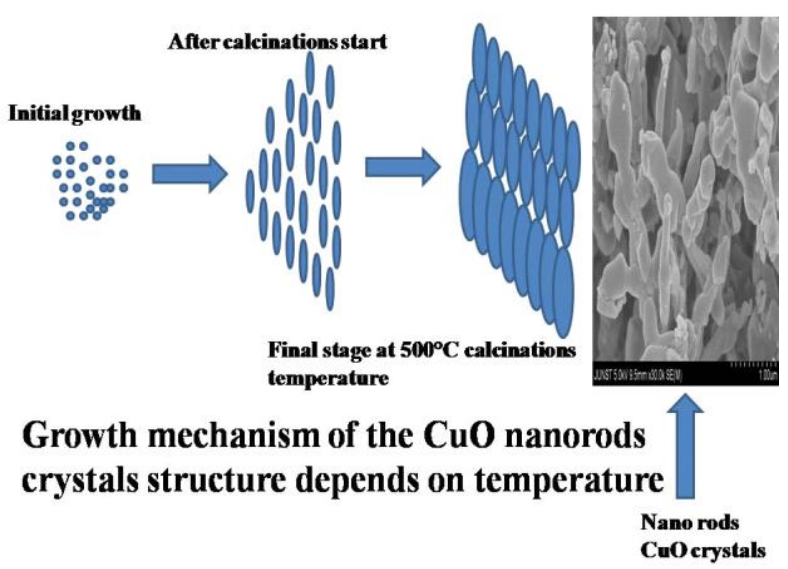

\section{FTIR Study}

FTIR is particularly useful for identification of different functional groups and compounds due to their absorption of energy in different range. FTIR used on the fact that the most molecules absorb light in the infra-red region of the electromagnetic spectrum. This absorption corresponds specifically to the bonds present in the molecule. The frequency ranges are measured as wave numbers typically over the range $400-3500 \mathrm{~cm}^{-1}$. [17]

The background emission spectrum of the IR source is first recorded, followed by the emission spectrum of the IR source with the sample in place. The ratio of the sample spectrum to the background spectrum is directly related to the sample's absorption spectrum. The resultant absorption spectrum from the bond natural vibration frequencies indicates the presence of various chemical bonds and functional groups present in the sample.

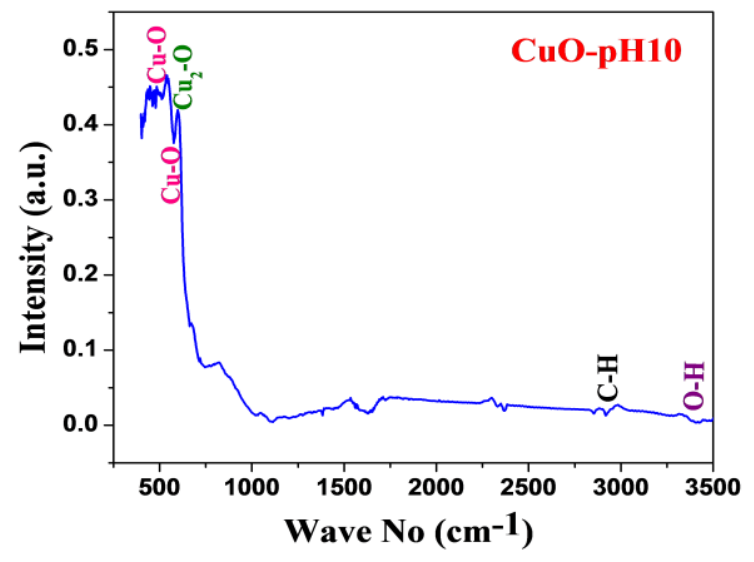

Fig 4 a) FTIR spectrum of $\mathrm{CuO}$ nano rods bond absorbtion

Fig 4 shows the FTIR spectra of $\mathrm{CuO}$ Nano rods powder absorption peak of 498 and 585 correspond to the characteristic stretching vibrations of $\mathrm{Cu}-\mathrm{O}$ bond in the monoclinic $\mathrm{CuO}$. And the absence of observation peak at 610 corresponding to the infrared active mode of confirms that synthesized product is pure $\mathrm{CuO}$ [18].

Absorption peak observed in the range between 2850-2950 corresponding to the anty-symmetric and symmetric vibration of $\mathrm{V}-\mathrm{H}$ bands. Also absorption peak observed in the range of 3300-3400 corresponds to stretching O-H bond.[15]

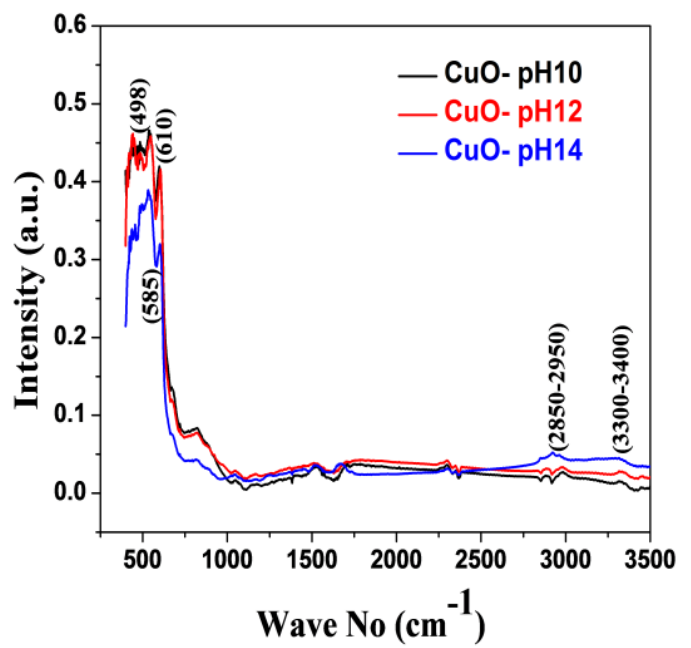

Fig 4 b) FTIR spectrum of $\mathrm{CuO}$ nanorods in the range between 400 3500

\section{Raman Analysis}

Raman spectroscopy, which is a sensitive probe to the local atomic arrangements and vibrations of the material, has been also widely used to investigate the microstructural nature of the Nano-sized materials in general and $\mathrm{CuO}$ nanomaterial in particular. Raman scattering also provides useful information about the structures and bonds of materials. Raman scattering also provides useful information about the existence of unintended phase such as $\mathrm{Cu}_{2} \mathrm{O}$ or $\mathrm{Cu}(\mathrm{OH})_{2}$ or show the crystallinity of the product.

The space group of $\mathrm{CuO}$ is with two molecules per primitive cell so the zone center Raman active normal modes of $\mathrm{CuO}$ are. Among these vibration modes, there are three acoustic modes (), six infrared active modes $\left(3 \mathrm{~A}_{\mathrm{u}}+3 \mathrm{~B}_{\mathrm{g}}\right)$, and three Raman active modes $\left(\mathrm{Ag}_{\mathrm{g}}+2 \mathrm{~B}_{\mathrm{g}}\right)$. Three well known bands of $\mathrm{CuO}$ are $\mathrm{Ag}_{\mathrm{g}}\left(296 \mathrm{~cm}^{-1}\right),\left(346 \mathrm{~cm}^{-1}\right)$ and $\left(631 \mathrm{~cm}^{-1}\right)$. Fig.5 Raman spectra of $\mathrm{CuO}$ nanostructures prepared by microwave irradiation method with three typical modes.

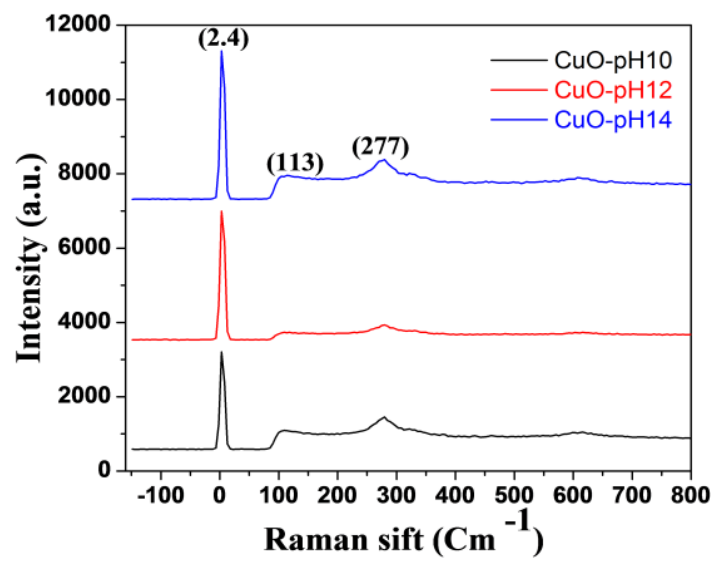

Fig 5 RAMAN Spectrum of CuO Nano Rods 
$\mathrm{Xu}$ et al. studied Raman spectra of $\mathrm{CuO}$ nanocrysrals with different grain size at room temperature and temperatures up to $873 \mathrm{~K}$. They reported that Raman intensity is related to the grain size. Samples of smaller grain sie show stronger and sharper Raman Peaks which also shift to smaller wavenumbers. The red shift could be explained by the phonon confinement effect in nanometer size materials.[6] It should be noted that crystal defects, of which number increase rapidly as the grain size decreases due to the large surface/volume ratio, could contribute significantlyto Raman spectra as all of three Raman modes in $\mathrm{CuO}$ relate only to the vibration of oxygen atoms as was pointed out by Irwin and Wei.

Apart from the three main vibration modes above, Wang et al. reported multiphonon band of $\mathrm{CuO}$ nanostructure, which appears at wavenumber of $1130 \mathrm{~cm}^{-1}$ and relates to the inharmonic coupling between phonons in polar solid. In particular, the multiphonon band $\mathrm{CuO}$ was suggested to be the stretching vibration in the - plane, induced by the electronic density variation in this layer.

The intensity of multiphonon Raman peak is much weaker than that of the one phonon band and varies with morphogy and the size of the as prepared nanostructures. The authors reported that the multiphonon band of the as prepared $\mathrm{CuO}$ nanostructures with belt-like morphology possesses higher intensity than that of the $\mathrm{CuO}$ nanostructures with stuttlelike morphology, while Raman intensity than that of $\mathrm{CuO}$ nanostructures with shuttle-like morphology, while Raman intensity of multiphonon band of the stuttle-like morphology is higher than that of the $\mathrm{CuO}$ nanostructures with bamboo leaf-like morphology.

The difference in the Raman intensity of different morphology was explained by anisotropy of different nanostructures. The electronic movement along the - plane becomes significant in - plane and promotes the intensity of $2 \mathrm{~B}_{\mathrm{g}}$ mode in belt-, stuttle-, and bamboo leaf-like nanostructures. Another expiation for the variation in the Raman intensity of this mode is the Phonon-Plasmon coupling due to high local density of anisotropic carriers in $\mathrm{CuO}$ nanostructures. The variation in the multiphonon intensity shows a finite size and crystallinity effect of $\mathrm{CuO}$ nanostructures.

\section{CONCLUSION}

In this study, $\mathrm{CuO}$ nanoparticles synthesized by the chemical precipitation method. Where $\mathrm{CuO}$ varies with different $\mathrm{pH}$ (power of hydrogen) values.

The structure and morphology of the nanoparticles charactized by XRD spectroscopy, and SEM technique. Morphology of $\mathrm{CuO}$ obtained monoclinic i.e. short nanotube. The crystal size of the $\mathrm{CuO}$ is $23 \mathrm{~nm}$.

From the FTIR spectrum with in the range (400-3500), find out the band stretching present in $\mathrm{CuO}$ material provided better confirmation of the material is formed. Raman spectra as all of the three Raman modes in $\mathrm{CuO}$ related only to the vibration of oxygen atoms.

After the numerous analysis such as marphology and crystalline of the material result are pretty good so Raman spectra conclude that improved optical and some electrical properties from this prepared sample are useful various applications in solar cells and capacitors and other devices i.e. semiconducting devices.

\section{REFERENCES}

[1] AndZayyoun, N., et al. "Effect of solvent on the morphological and optical properties of $\mathrm{CuO}$ nanoparticles prepared by simple sol-gel process." J. Mater. Environ. Sci. 7.5 (2016): 1791-1797.

[2] Wu, Jianghong, et al. "Controllable hydrothermal synthesis of MnO 2 nanostructures." (2013)

[3] Jankovský, Ondřej, et al. "Synthesis of MnO, Mn 2 O 3 and Mn 3 O 4 nanocrystal clusters by thermal decomposition of manganese glycerolate." Ceramics International 41.1 (2015): 595-601.

[4] Bibi, Maryam, Hussain Abbas, and Sabah Baqi. "Outcome of temperature variation on sol-gel prepared $\mathrm{CuO}$ nanostructure properties (optical and dielectric)." Materials Chemistry and Physics 192 (2017): 67-71..

[5] Zhang, Qiaobao, et al. "CuO nanostructures: synthesis, characterization, growth mechanisms, fundamental properties, and applications." Progress in Materials Science 60 (2014): 208-337.

[6] Kumar, Harish, and Sangwan P. Manisha. "Synthesis and characterization of $\mathrm{MnO} 2$ nanoparticles using co-precipitation technique." Int J Chem Chem Eng 3.3 (2013): 155-60.

[7] Qian, Yong, et al. "Synthesis of cuprous oxide (Cu2O) nanoparticles/graphene composite with an excellent electrocatalytic activity towards glucose." Int. J. Electrochem. Sci 7 (2012): 10063-10073.

[8] Radhakrishnan, A. Asha, and B. Baskaran Beena. "Structural and optical absorption analysis of $\mathrm{CuO}$ nanoparticles." Indian Journal of Advances in Chemica l Science 2 (2014): 158-161.

[9] Srivastava, Sanjay. "Synthesis and characterisation of copper oxide nanoparticles." IOSR Journal of Applied Physics 5 (2013): 61-65.

[10] Kannaki, K., P. S. Ramesh, and D. Geetha. "Hydrothermal synthesis of $\mathrm{CuO}$ nanostructure and their characterizations." IJSER 3.9 (2012): 1-4

[11] Varughese, G., et al. "Characterisation and optical studies of copper oxide nanostructures doped with lanthanum ions." Advances in Materials Science 14.4 (2014): 49-60.

[12] Nithya, K., et al. "Preparation and Characterization of Copper Oxide Nanoparticles." Int. J. ChemTech Res 6 (2014): 2220-2222.

[13] Phiwdang, Kankanit, et al. "Synthesis of $\mathrm{CuO}$ nanoparticles by precipitation method using different precursors." Energy Procedia 34 (2013): 740-745.

[14] Kayani, Zohra Nazir, et al. "Characterization of Copper Oxide Nanoparticles Fabricated by the Sol-Gel Method." Journal of Electronic Materials 44.10 (2015): 3704.

[15] Arockiasamy, Joseph Sagaya Kennedy, and Johnson Irudayaraj. "Natural dye sensitized $\mathrm{CuO}$ nanorods for luminescence applications." Ceramics International 42.5 (2016): 6198-6205.

[16] Tran, Thi Ha, and Viet Tuyen Nguyen. "Copper oxide nanomaterials prepared by solution methods, some properties, and potential applications: A brief review." International Scholarly Research Notices 2014 (2014).

[17] Davar, F., Feresheh Z. and Salavati-Niasari, M., 2009 Nanoparticles $\mathrm{Ni}$ and $\mathrm{NiO}$; synthesis, characterization and magnetic properties. Journal of Alloys and Compounds, 476(1),pp.797-801 .

[18] Li, M., Dong, B., Gao, G. and Ding, S., 2015. Synthesis of nickel oxide/reduced grapheme oxide composite with nanosheet-on-sheet nanostructure for lithium-ion batteries. Materials Letters, 155, pp.30-33. 